Pacific Journal of Mathematic 


\title{
ON THE EQUATION $\varphi(x)=\int_{x}^{x+1} K(\xi) f[\varphi(\xi)] d \xi$
}

\author{
Morton I. Slater
}

Suppose $K(x)$ measurable and $0<K(x) \leqq 1$ for $x \in(-\infty, \infty)$. Suppose $f(u)$ convex for $u \in[0,1], f(0)=0, f(u)>0$ for $u \in(0,1)$, and $f(u)=1-f^{\prime}(1)(1-u)+O(1-u)^{1+\delta}$ as $u \rightarrow 1$ for some $\delta>0$. (Example : $f(u)=u^{p}, p \geqq 1$.)

Theorem : The equation $\left(^{*}\right) \varphi(x)=\int_{x}^{x+1} K(\xi) f[\varphi(\xi)] d \xi$ has a solution $\varphi(x)$ satisfying $0<\varphi(x) \leqq 1$ for $x \in(-\infty, \infty)$ if and only if $\int^{\infty} e^{\alpha x}[1-K(x)] d x<\infty$ where $\alpha$ is the largest real root of $\alpha=$ $f^{\prime}(1)\left(1-e^{-\alpha}\right)$. Furthermore, if $\varphi$ is any such solution of $\left(^{*}\right)$, then the limits $\varphi( \pm \infty)$ exist and satisfy

$$
\frac{\varphi(+\infty)-\varphi(-\infty)}{2}=\int_{-\infty}^{\infty}[\varphi(x)-K(x) f[\varphi(x)]] d x
$$

In 1960 M. L. Slater and H. S. Wilf [2] studied the linear integral equation $\varphi(x)=\int_{x}^{x+1} K(\xi) \varphi(\xi) d \xi,-\infty<x<\infty$, with $\varphi(+\infty)=1$, and obtained the following results. Under the assumptions $1^{\circ} K(x)$ measurable, $2^{\circ} 0<K(x) \leqq 1,3^{\circ} K(x)$ increasing for sufficiently large $x$, and $4^{\circ} \lim _{x \rightarrow \infty} K(x)=1$, a solution $\varphi$ of the equation exists satisfying $\varphi(+\infty)=1$ if and only if $\int^{\infty}[1-K(x)] d x<\infty$. (We use the notation " $\int^{\infty}$ " to mean "the integral from any finite limit to infinity.") If in addition $5^{\circ}$

$$
\lim _{x \rightarrow-\infty} \int_{x}^{x+1}|K(\xi+1)-K(\xi)| d \xi=0
$$

then $\varphi(-\infty)$ exists.

The purpose of this paper is to extend the above results in two directions; namely to generalize the equation and to remove some of the restrictions on $K(x)$.

Accordingly, we consider throughout the paper the equation

$$
\varphi(x)=\int_{x}^{x+1} K(\xi) f[\varphi(\xi)] d \xi
$$

with the requirement that the solution $\varphi$ satisfy $0<\varphi(x) \leqq 1$ for all $x$. The functions $f(u)=u^{p}, p \geqq 1$, were the prototypes for the analysis and the results which we summarize below are valid for at least these functions. However, for each theorem of the paper a wider class of functions $\{f\}$ is specified in order to clarify the logical structure of the result. The weakening of the restrictions on $K(x)$ 
is easily stated. Assumptions $3^{\circ}, 4^{\circ}$, and $5^{\circ}$ are dropped completely and without replacement.

In $\S$ II we consider the question of existence of the limits $\varphi( \pm \infty)$. Theorem 1 and its corollary establish that under conditions $1^{\circ}$ and $2^{\circ}$ both of the limits exist. (The order argument used in $\S$ II was already used to some extent in [2].)

Section III contains the proofs of two lemmas required for the main existence theorem-Theorem 2 in $\S$ IV. This theorem provides a necessary and sufficient condition for the existence of a solution of the required type. The condition reduces in the linear case to that obtained in [2]. The underlying assumptions on $K$ are again only $1^{\circ}$ and $2^{\circ}$.

Section $\mathrm{V}$ contains an extension of an integral relation proved in [2] (Theorem 3), and $\S$ VI gives a brief discussion of the actual range of validity of the results (Theorem 4).

\section{Existence of $\varphi( \pm \infty)$.}

THEOREM 1. Suppose $K(x)$ measurable and $0<K(x) \leqq 1$ a.e. for $-\infty<x<\infty$, and suppose $\varphi(x)$ satisfies $0<\varphi(x) \leqq 1$ and the linear equation

$$
\varphi(x)=\int_{x}^{x+1} K(\xi) \varphi(\xi) d \xi
$$

for all $x$. Then both $\varphi(+\infty)$ and $\varphi(-\infty)$ exist and satisfy

$$
\frac{\varphi(+\infty)-\varphi(-\infty)}{2}=\int_{-\infty}^{\infty} \varphi(\xi)[1-K(\xi)] d \xi \text {. }
$$

Proof. Define

$$
\begin{aligned}
& G(x)=\int_{0}^{1} K(x+1-y) \varphi(x+1-y) y d y . \\
& G(x)=\int_{x}^{x+1} K(\xi) \varphi(\xi)(x+1-\xi) d \xi
\end{aligned}
$$

is absolutely continuous over any finite interval, and, by using equation (1), one can verify that $G^{\prime}(x)=\varphi(x)[1-K(x)]$ a.e. Thus $G(x)$ is increasing so that $G( \pm \infty)$ exist, are finite, and

$$
\infty>G(+\infty)-G(-\infty)=\int_{-\infty}^{\infty} \varphi(x)[1-K(x)] d x .
$$

We first prove $\varphi(+\infty)$ exists. Set $M=\lim \sup _{x \rightarrow \infty} \varphi(x), \quad m=\lim$ $\inf _{x \rightarrow \infty} \varphi(x)$, and suppose $M>m$. Set 


$$
k=\lim _{x \rightarrow \infty} \sup \int_{x}^{x+1}\left|\varphi^{\prime}(\xi)\right| d \xi
$$

Almost everywhere,

$$
\varphi^{\prime}(x)=\varphi(x)[1-K(x)]-\varphi(x+1)[1-K(x+1)]+\varphi(x+1)-\varphi(x),
$$

so that since

$$
\infty>\int_{-\infty}^{\infty} \varphi[1-K] d x, \quad k \leqq M-m .
$$

Now, it follows from equation (1) that $\varphi$ cannot have a proper maximum at the left hand endpoint of an interval of length one; that is, it is impossible that for any $x, \varphi(x)>\varphi(y)$ for all $y$ satisfying $x<$ $y \leqq x+1$. We shall use this fact (which we shall refer to as the "proper maximum property") to show that given any positive $\varepsilon<$ $(M-m) / 2$ and $X$ arbitrarily large, there exist triples $x, y, z$ satisfying $X<x<y<z$, and $z-x \leqq 1$, for which $\varphi(x)=\varphi(z)=M-\varepsilon$, and $\varphi(y)=m+\varepsilon$.

Choose $x_{0}>X$ so that $\varphi\left(x_{0}\right)=M-\varepsilon$ and let $y$ be the first point greater than $x_{0}$ at which $\varphi(y)=m+\varepsilon$. Now let $x$ be the largest point less than $y$ at which $\varphi(x)=M-\varepsilon . \quad y-x<1$; otherwise the proper maximum property would be violated. Finally let $z$ be the first point greater than $y$ at which $\varphi(z)=M-\varepsilon$. $z-x \leqq 1$ for the same reason.

Given $\varepsilon>0$, choose $X=X(\varepsilon)$ so that for all

$$
x \geqq X, \quad k+\varepsilon>\int_{x}^{x+1}\left|\varphi^{\prime}(\xi)\right| d \xi .
$$

Now choose $x, y, z$ as described in the preceding paragraph using $X=X(\varepsilon)$. Then

$$
\begin{aligned}
k+\varepsilon & >\int_{x}^{z}\left|\varphi^{\prime}(\xi)\right| d \xi \\
& \geqq\left|\int_{x}^{y} \varphi^{\prime}(\xi) d \xi\right|+\left|\int_{y}^{z} \varphi^{\prime}(\xi) d \xi\right| \\
& =2(M-m-2 \varepsilon) . \text { Hence } \\
k & \geqq 2(M-m), \text { contradicting } k \leqq M-m .
\end{aligned}
$$

Thus $M=m=\varphi(+\infty)$, and incidentally, $k=0$.

The proof that $\varphi(-\infty)$ exists is similar to the preceding proof. Define $M, m$, and $k$ as above but with respect to $-\infty$. Then as in the previous case, $k \leqq M-m$. To find the appropriate triples to complete the proof, we proceed slightly differently. Given $X$ choose $y<X-1$ such that $\varphi(y)=m+\varepsilon$. Then take $x$ to be the first point less than 
$y$ at which $\varphi(x)=M-\varepsilon$ and $z$ to be the first point greater than $y$ at which $\varphi(z)=M-\varepsilon$. (The existence of such a $z$ is guaranteed by the proper maximum property.) The remainder of the proof is identical to the corresponding part of the preceding proof.

$G( \pm \infty)$ can be evaluated in terms of $\varphi( \pm \infty)$, yielding the integral formula obtained in [2]. For, using equation (1) and an interchange of the order of integration, we obtain

$$
\int_{x}^{x+1} G(\xi) d \xi=\int_{0}^{1} \varphi(x+1-y) y d y .
$$

Hence

$$
G( \pm \infty)=\frac{\varphi( \pm \infty)}{2}
$$

and so

$$
\int_{-\infty}^{\infty} \varphi[1-K] d \xi=\frac{\varphi(+\infty)-\varphi(-\infty)}{2}
$$

Corollary. Suppose $f(u)$ is continuous and satisfies $0<f(u) \leqq$ $u$ for $u \in(0,1]$, suppose $K(x)$ is measurable and satisfies $0<K(x) \leqq 1$ for $-\infty<x<\infty$, and suppose $\varphi(x)$ satisfies $0<\varphi(x) \leqq 1$ and the equation

$$
\varphi(x)=\int_{x}^{x+1} K(\xi) f[\varphi(\xi)] d \xi
$$

over the same range of $x$. Then both $\varphi(+\infty)$ and $\varphi(-\infty)$ exist.

Proof. Apply Theorem 1 to $K f[\varphi] / \varphi$ in place of $K$.

\section{The main lemmas.}

Lemma 1. Suppose $X \in(-\infty, \infty), a \geqq 1$, and $\mu_{0}(x)$ measurable, $0 \leqq \mu_{0}(x)<\infty$, for $x \geqq X$. Then the linear integral inequality

$$
\mu(x) \geqq \mu_{0}(x)+a \int_{x}^{x+1} \mu(\xi) d \xi
$$

has a solution $\mu(x)$ with $0 \leqq \mu(x)<\infty$ for $x \geqq X$ if and only if

$$
\int^{\infty} e^{\alpha x} \mu_{0}(x) d x<\infty,
$$

where $\alpha=\alpha(a)$ is the largest real root of $\alpha=a\left(1-e^{-\alpha}\right)$. (Note that $\alpha>0$ if $a>1$ and $\alpha=0$ if $a=1$.) Furthermore, if a finite nonnegative solution of $\left(^{*}\right)$ exists, then there is also such a solution of 
(*) with the inequality replaced by equality which has the additional property that $\lim _{x \rightarrow \infty}\left[\mu(x)-\mu_{0}(x)\right]=0$.

Proof. Let $\mu(x)$ be a finite nonnegative solution of $\left(^{*}\right)$. Let $F(x)$ be any increasing continuously differentiable function defined for $x \geqq X-1$. Then for $x \geqq X$

$$
\begin{aligned}
& \frac{d}{d x} \int_{0}^{1} \mu(x+1-y)[F(x)-F(x-y)] d y \\
& \quad=F^{\prime}(x) \int_{0}^{1} \mu(x+1-y) d y+\mu(x)[F(x-1)-F(x)] \\
& \quad \leqq \mu(x)\left[\frac{F^{\prime}(x)}{a}+F(x-1)-F(x)\right]-\frac{\mu_{0}(x) F^{\prime}(x)}{a} .
\end{aligned}
$$

If $a>1$, set $F(x)=\left(e^{\alpha x}-1\right) / \alpha$, where $\alpha$ is defined above, and if $a=1$ set $F(x)=x$, the limiting value as $\alpha$ approaches zero. The expression in square brackets vanishes, and we have

$$
\frac{d}{d x} \int_{0}^{1} \mu(x+1-y)[F(x)-F(x-y)] d y \leqq-\frac{\mu_{0}(x) F^{\prime}(x)}{a}
$$

Thus, since $\mu(x) \geqq 0$, we find

$$
\int_{x}^{\infty} \mu_{0}(\xi) F^{\prime}(\xi) d \xi \leqq a \int_{0}^{1} \mu(x+1-y)[F(x)-F(x-y)] d y,
$$

thereby establishing necessity.

To prove sufficiency we first define

$$
\begin{array}{crr}
\gamma(u) & =a e^{-\alpha u} & 0 \leqq u \leqq 1, \\
& =0 & u>1,
\end{array}
$$

and show that the solution $\nu(u)$ of the equation

$$
\nu(u)=\gamma(u)+\int_{0}^{u} \nu(v) \gamma(u-v) d v
$$

is unique, nonnegative, and bounded. Equation (7) is an example of a renewal equation, and uniqueness and nonnegativity follow from the general theory of such equations. (See for example Doetsch [1], Volume III, page 145, Theorem I.) Boundedness, which is essential here, can be shown by noting that if $\nu$ is unbounded then there is a $\bar{u}>1$ such that if $u<\bar{u}$ then $\nu(u)<\nu(\bar{u})$. But

$$
\nu(\bar{u})=\int_{\bar{u}_{-1}}^{\bar{u}} \nu(v) \gamma(\bar{u}-v) d v,
$$

and since $\int_{0}^{1} \gamma(v) d v=1$ (a consequence of $\alpha=\alpha(\alpha)$ ), 


$$
\int_{\bar{u}-1}^{\bar{u}}[\nu(\bar{u})-\nu(v)] \gamma(\bar{u}-v) d v=0,
$$

contradicting the positivity of $\gamma(u)$.

We now proceed with the proof of sufficiency and show that

$$
\mu(x)=\mu_{0}(x)+\int_{0}^{\infty} \nu(u) \mu_{0}(x+u) e^{\alpha u} d u
$$

is a solution of $\left({ }^{*}\right)$. Actually we show that $\mu(x)$ satisfies $\left({ }^{*}\right)$ with equality. To do this we must verify that

$$
\int_{0}^{\infty} \nu(u) e^{\alpha u} \mu_{0}(x+u) d u=a \int_{x}^{x+1} \mu(\xi) d \xi .
$$

The right hand side of (9) can be rewritten as

$$
\int_{0}^{1} a e^{-\alpha u} e^{\alpha u} \mu(x+u) d u=\int_{0}^{\infty} \gamma(u) e^{\alpha u} \mu(x+u) d u,
$$

and substituting (8) this becomes

$$
\int_{0}^{\infty} \gamma(u) e^{\alpha u} \mu_{0}(x+u) d u+\int_{0}^{\infty} \int_{0}^{\infty} \nu(v) \gamma(u) e^{\alpha(u+v)} \mu_{0}(x+u+v) d u d v .
$$

If in the double integral we set $u+v=w$ and $v=z$ and integrate first with respect to $z$ we obtain

$$
\int_{0}^{\infty} d w e^{\alpha w} \mu_{0}(x+w) \int_{0}^{w} \nu(z) \gamma(w-z) d z .
$$

Thus, after renaming variables, the right side of (9) becomes

$$
\int_{0}^{\infty} d u e^{\alpha u} \mu_{0}(x+u)\left\{\gamma(u)+\int_{0}^{u} \nu(v) \gamma(u-v) d v\right\},
$$

and the required equality is a consequence of (7).

To prove the last statement of the lemma we show now that

$$
\lim _{x \rightarrow \infty} \int_{0}^{\infty} \nu(u) \mu_{0}(x+u) e^{\alpha u} d u=0 .
$$

This follows from the boundedness of $\nu(u)$ and the fact that

$$
\int^{\infty} e^{\alpha x} \mu_{0}(x) d x<\infty \text {. }
$$

Lemma 2. Suppose $a>1$ and $\alpha=\alpha(a)$ is the largest real root of $\alpha=a\left(1-e^{-\alpha}\right)$. Then for all $\beta<\alpha \int^{\infty} e^{\beta x} \mu(x) d x<\infty$, where $\mu(x)$ is any nonnegative finite-valued solution of $\left(^{*}\right)$ with the parameter a. 


$$
\frac{d}{d x}\left[e^{\alpha x} \int_{0}^{1} \mu(x+1-y)\left(1-e^{-\alpha y}\right) d y\right] \leqq 0 .
$$

Hence for some nonnegative $A, \int_{0}^{1} \mu(x+1-y)\left(1-e^{-\alpha y}\right) d y \leqq A e^{-\alpha x}$, and

$$
\begin{aligned}
\frac{A}{\alpha-\beta} e^{-(\alpha-\beta) x} & \geqq \int_{x}^{\infty} e^{\beta \xi} d \xi \int_{0}^{1} \mu(\xi+1-y)\left(1-e^{-\alpha y}\right) d y \\
& =\int_{0}^{1} e^{-\beta(1-y)}\left(1-e^{-\alpha y}\right) d y \int_{x}^{\infty} e^{\beta(\xi+1-y)} \mu(\xi+1-y) d \xi \\
& \geqq C \int_{x+1}^{\infty} e^{\beta \xi} \mu(\xi) d \xi, \text { where } C=\int_{0}^{1} e^{-\beta(1-y)}\left(1-e^{-\alpha y}\right) d y .
\end{aligned}
$$

\section{Existence of solutions.}

THEOREM 2. Suppose $K(x)$ measurable and $0<K(x) \leqq 1$ a.e. in $-\infty<x<+\infty$. Suppose $f(u)$ convex for $0 \leqq u \leqq 1, f(0)=0, f(1)=1$, $f(u)>0$ for $0<u<1, f^{\prime}(1)<\infty$, and $f(u)=1-f^{\prime}(1)(1-u)+$ $O(1-u)^{1+\delta}$ as $u \rightarrow 1$ for some $\delta>0$. Then the equation

$$
\varphi(x)=\int_{x}^{x+1} K(\xi) f[\varphi(\xi)] d \xi
$$

has a solution $\varphi(x),-\infty<x<\infty$, satisfying $0<\varphi(x) \leqq 1$, if and only if

$$
\int^{\infty} e^{\alpha \xi}(1-K(\xi)) d \xi<\infty,
$$

where $\alpha=\alpha\left(f^{\prime}(1)\right)$ is the largest real root of $\alpha=f^{\prime}(1)\left(1-e^{-\alpha}\right)$. If $f^{\prime}(1)>1$, then $1-\varphi(x)=O\left(e^{-\beta x}\right)$ as $x \rightarrow \infty$ for all $\beta<\alpha=\alpha\left(f^{\prime}(1)\right)$.

Sufficiency. Define

$$
\varphi_{0}(x) \equiv 1, \varphi_{n+1}(x)=\int_{x}^{x+1} K(\xi) f\left[\varphi_{n}(\xi)\right] d \xi .
$$

Then, since $f(x)$ is increasing, $0<\varphi_{n+1}(x) \leqq \varphi_{n}(x)$ for all $x$ and $n \geqq 0$. Thus $\lim _{n \rightarrow \infty} \varphi_{n}(x)=\varphi(x)$ exists and $\varphi(x)$ satisfies equation (10) by the dominated convergence theorem. We must show that $\varphi(x)$ is positive. For $n \geqq 1$

$$
\begin{aligned}
\varphi_{n}(x)-\varphi_{n+1}(x) & =\int_{x}^{x+1} K(\xi)\left[f\left(\varphi_{n-1}\right)-f\left(\varphi_{n}\right)\right] d \xi \\
& \leqq f^{\prime}(1) \int_{x}^{x+1}\left[\varphi_{n-1}(\xi)-\varphi_{n}(\xi)\right] d \xi
\end{aligned}
$$

Thus 


$$
\begin{aligned}
1-\varphi_{n+1}(x) & \leqq 1-\varphi_{1}(x)+f^{\prime}(1) \int_{x}^{x+1}\left[1-\varphi_{n}(\xi)\right] d \xi \\
& =\int_{x}^{x+1}[1-K(\xi)] d \xi+f^{\prime}(1) \int_{x}^{x+1}\left[1-\varphi_{n}(\xi)\right] d \xi .
\end{aligned}
$$

Since

$$
\int^{\infty} e^{\alpha x} \int_{x}^{x+1}[1-K(\xi)] d \xi d x<\infty
$$

since $f^{\prime}(1) \geqq 1$, and since

$$
\lim _{x \rightarrow \infty} \int_{x}^{x+1}(1-K) d \xi=0
$$

there is by Lemma 1 a nonnegative function $\mu(x)$ satisfying

$$
\mu(x)=\int_{x}^{x+1}[1-K(\xi)] d \xi+f^{\prime}(1) \int_{x}^{x+1} \mu(\xi) d \xi \text { and } \lim _{x \rightarrow \infty} \mu(x)=0 .
$$

Now

$$
1-\phi_{1}(x)=\int_{x}^{x+1}[1-K(\xi)] d \xi \leqq \mu(x)
$$

and by induction using (11) and (12) we see that $1-\varphi_{n}(x) \leqq \mu(x)$ and consequently $1-\varphi(x) \leqq \mu(x)$. Thus $\lim _{x \rightarrow \infty} \varphi(x)=1$, and if $\varphi(x)$ $=0$ for some $x$, there must be a largest $x$ at which $\varphi$ vanishes. But this clearly contradicts the fact that $\varphi$ is a solution of (10).

Necessity. Suppose that $\varphi(x)$ is a solution of the required type. By the corollary to Theorem $1, \varphi(+\infty)$ exists. Now, in fact, $\varphi(+\infty)=$ lub $\varphi(x)$, for if not there would exist an $\bar{x}$ such that for all $x>\bar{x}$, $\varphi(\bar{x})>\varphi(x)$, which would contradict the fact that $\varphi(x)$ satisfies $(10)$. In particular this means that $\varphi(+\infty)>0$. If $f(u) \equiv u$, then $\varphi(x) / \varphi(+\infty)$ is a solution whose limit at infinity is one. If $f(u) \not \equiv u$, then $f(u)<u$ for $0<u<1$, and from (10) we see that since $\varphi(+\infty) \neq 0$, it mus'; be equal to one. Thus we may always assume $\varphi(+\infty)=1$.

Writing $f(u)=1-f^{\prime}(1)(1-u)+R(u)$ we have

$$
\begin{aligned}
1-\varphi(x)= & \int_{x}^{x+1}[1-K(\xi)]\left[1-f^{\prime}(1)(1-\varphi(\xi))\right] d \xi \\
& -\int_{x}^{x+1} K(\xi) R[\varphi(\xi)] d \xi+f^{\prime}(1) \int_{x}^{x+1}(1-\varphi(\xi)) d \xi .
\end{aligned}
$$

If $f(u) \equiv u$, then $R(u) \equiv 0$ and $f^{\prime}(1)=1$ so that the use of Lemma 1 with $\mu(x)=1-\varphi(x)$ allows one to conclude that

$$
\int^{\infty} d x \int_{x}^{x+1}[1-K(\xi)] \varphi(\xi) d \xi<\infty .
$$


Then, since $\varphi(+\infty)=1$, we obtain the desired result that

$$
\int^{\infty}[1-K(\xi)] d \xi<\infty .
$$

If $f(u) \neq u$, then $f^{\prime}(1)>1$. We first show that if $\delta>0$, then

$$
\int^{\infty} e^{\alpha \xi}[1-\varphi(\xi)]^{1+\delta} d \xi<\infty \text {. }
$$

Define

$$
g(x)=\int_{0}^{1}\{1-K(x+1-y) f[\varphi(x+1-y)]\} y d y .
$$

Now $g(x)$ is absolutely continuous over any finite interval and since for almost all $x, g^{\prime}(x)=-[\varphi(x)-K(x) f[\varphi(x)]] \leqq 0, g(x)$ is decreasing. Furthermore from (10)

$$
\int_{x}^{x+1} g(\xi) d \xi=\int_{0}^{1}[1-\varphi(x+1-y)] y d y .
$$

Thus for any $\varepsilon \in\left(0, f^{\prime}(1)-1\right)$ and for sufficiently large $x$, since $\varphi(+\infty)=1$, we have $1-f[\varphi(x)] \geqq\left(f^{\prime}(1)-\varepsilon\right)(1-\varphi(x))$, so that

$$
\begin{aligned}
\int_{x}^{x+1} g(\xi) d \xi & \leqq \frac{1}{f^{\prime}(1)-\varepsilon} \int_{0}^{1}\{1-f[\varphi(x+1-y)]\} y d y \\
& \leqq \frac{g(x)}{f^{\prime}(1)-\varepsilon} .
\end{aligned}
$$

Hence by Lemma 2,

$$
\int^{\infty} e^{\beta x} g(x) d x<\infty \text { for all } \beta<\alpha=\alpha\left(f^{\prime}(1)\right) .
$$

Since $g(x)$ is decreasing,

$$
g(x+1) e^{\beta x} \leqq \int_{x}^{x+1} e^{\beta \xi} g(\xi) d \xi<A=A(\beta),
$$

and so $g(x)=O\left(e^{-\beta x}\right)$ for all $\beta<\alpha$. On the other hand

$$
\begin{aligned}
1-\varphi(x) & =\int_{x}^{x+1}\{1-K(\xi) f[\varphi(\xi)]\} d \xi \\
& =\int_{0}^{1}\{1-K(x+1-y) f[\varphi(x+1-y)]\} d y \\
& \leqq 2 g(x)+2 g(x+1 / 2)=O\left(e^{-\beta x}\right),
\end{aligned}
$$

so that if we now choose $\beta$ so that $\beta(1+\delta)>\alpha$, we have the required result.

Since $R(\varphi)$ by hypothesis is $O\left\{(1-\varphi)^{1+\delta}\right\}$, the equation 


$$
\mu(x)=\int_{x}^{x+1} K(\xi) R[\varphi(\xi)] d \xi+f^{\prime}(1) \int_{x}^{x+1} \mu(\xi) d \xi,
$$

has by Lemma 1 a nonnegative solution $\mu(x)$ for which $\lim _{x \rightarrow \infty} \mu(x)=0$. $(R(\varphi) \rightarrow 0$.) Now,

$$
\varphi(x)=\int_{x}^{x+1} K(\xi) R(\varphi) d \xi+\int_{x}^{x+1} K(\xi)\left[1-f^{\prime}(1)(1-\varphi(\xi))\right] d \xi .
$$

Define $\psi_{0}(x)=\varphi(x)$, and for $n \geqq 0$,

$$
\psi_{n+1}(x)=\int_{x}^{x+1} K(\xi)\left[1-f^{\prime}(1)\left(1-\psi_{n}(\xi)\right)\right] d \xi .
$$

Since $R(\varphi) \geqq 0$ (by the convexity of $f$ ), $\varphi(x)=\psi_{0}(x) \geqq \psi_{1}(x)$, and we see by induction using (14) that each $\psi_{n}(x) \geqq \psi_{n+1}(x)$. Thus $\varphi(x)-$ $\psi_{n}(x)$ is increasing with respect to $n$. Again,

(15) $\varphi(x)-\psi_{n+1}(x)=\int_{x}^{x+1} K(\xi) R(\varphi) d \xi+f^{\prime}(1) \int_{x}^{x+1} K(\xi)\left[\varphi(\xi)-\psi_{n}(\xi)\right] d \xi$.

Now, $\varphi(x)-\psi_{0}(x)=0 \leqq \mu(x)$, and by a second induction using (13) and (15) we see that $\varphi(x)-\psi_{n}(x) \leqq \mu(x)$. Thus $\psi_{n} \downarrow_{n} \psi(x)$ (say) satisfying $\varphi(x) \geqq \psi(x) \geqq \varphi(x)-\mu(x)$, and

$$
\psi(x)=\int_{x}^{x+1} K(\xi)\left[1-f^{\prime}(1)(1-\psi(\xi))\right] d \xi
$$

We rewrite (16) as

$$
\begin{aligned}
1-\psi(x)= & \int_{x}^{x+1}[1-K(\xi)]\left[1-f^{\prime}(1)(1-\psi(\xi))\right] d \xi \\
& +f^{\prime}(1) \int_{x}^{x+1}[1-\psi(\xi)] d \xi
\end{aligned}
$$

and note that since $\lim _{x \rightarrow \infty} \mu(x)=0$ there is an $X=X(\varepsilon)$ such that for $x \geqq X, 0 \leqq 1-\psi(x) \leqq \varepsilon$. Thus

$$
1-\psi(x) \geqq\left(1-f^{\prime}(1) \varepsilon\right) \int_{x}^{x+1}[1-K(\xi)] d \xi+f^{\prime}(1) \int_{x}^{x+1}[1-\psi(\xi)] d \xi,
$$

and so by Lemma 1,

$$
\int^{\infty} e^{\alpha \xi}[1-K(\xi)] d \xi<\infty
$$

V. An integral relation. Suppose $f(u)$ is as in Theorem 2 and in addition $f(u) \not \equiv u$. Then $\varphi(+\infty)=1$ and from equation (10) we see that $\varphi(-\infty)=0$ or 1 . Apply Theorem 1 with $K$ replaced by $K f(\varphi) / \varphi$. Then equation (2) becomes 


$$
\frac{1-\varphi(-\infty)}{2}=\int_{-\infty}^{\infty}\{\varphi(\xi)-K(\xi) f[\varphi(\xi)]\} d \xi \text {. }
$$

If $\varphi(-\infty)=1$, then $\varphi(x)=K(x) f[\varphi(x)]$ for almost all $x$, and since $\varphi>0$, this means that $\varphi \equiv 1$ and $K \equiv 1$ a.e. This yields the following relation.

Theorem 3. Let $f$ and $K$ be as in Theorem 2 and in addition assume $f(u) \neq u$ and $K(x) \not \equiv 1$ a.e. Then a solution $\varphi$ of equation (10) satisfies

$$
\int_{-\infty}^{\infty}\{\varphi(\xi)-K(\xi) f[\varphi(\xi)]\} d \xi=1 / 2 \text {. }
$$

VI. Concluding remarks. The hypotheses in Theorem 2 were chosen to make, in some sense, a "clean" theorem, and as is usually the case more is actually proved than is stated. Thus in proving sufficiency, no use is made of the assumption $R(u)=O(1-u)^{1+\delta}$. Furthermore very weak use is made of the convexity of $f$ and, in fact, the behavior of $f(u)$ in the neighborhood of $u=1$ is all that is significant in the following sense.

THEOREM 4. Let $\mathfrak{F}$ be the class of increasing, nonnegative, continuous functions defined on the unit interval such that if $f \in \mathfrak{F}$, then $f(1)=1$. Suppose that for a certain $f_{1} \in \mathfrak{F}$ equation (10) has a nonnegative solution $\varphi_{1}$ satisfying $\varphi_{1} \leqq 1$ and $\varphi_{1}(+\infty)=1$. Then if some other $f_{2} \in \widetilde{F}$ coincides with $f_{1}$ in some neighborhood of 1 , equation (10) with $f=f_{2}$ has a nonnegative solution $\varphi_{2}$ satisfying $\varphi_{2} \leqq 1$ and $\varphi_{2}(+\infty)=1$.

Proof. Suppose $f_{1}(u)=f_{2}(u)$ for $u_{0} \leqq u \leqq 1$. There is an $X$ such that for $x \geqq X, \varphi_{1}(x) \geqq u_{0}$. Set $\psi_{0}(x)=0$ for $x<X$ and $\psi_{0}(x)=\varphi_{1}(x)$ for $x \geqq X$. Then for $-\infty<x<+\infty$

$$
\psi_{0}(x) \leqq \int_{x}^{x+1} K(\xi) f_{2}\left[\psi_{0}(\xi)\right] d \xi
$$

Now for $n \geqq 0$ define

$$
\psi_{n+1}(x)=\int_{x}^{x+1} K(\xi) f_{2}\left[\psi_{n}(\xi)\right] d \xi
$$

Since $f_{2}$ is increasing, $\psi_{n+1}(x) \geqq \psi_{n}(x)$ for all $n$ and $x$ and in addition $\psi_{n}(x) \leqq 1$. Thus $\psi_{n}(x) \uparrow_{n} \varphi_{2}(x)$, a solution with $f=f_{2}$.

\section{ReFERENCES}

1. G. Doetsch, Handbuch Der Laplace-Transformation, Verlag Birkhauser, Basel, 1950-1956. 
2. M. L. Slater and H. S. Wilf, A class of linear differential-difference equations, Pacific J. Math. 104 (1960), 1419-1427.

Received June 7, 1965. This work was supported by the United States Atomic Energy Commission. Reproduction in whole or in part is permitted for any purpose of the U. S. Government.

SANDIA LABORATORY

Albuquerque, NeW Mexico 


\section{PACIFIC JOURNAL OF MATHEMATICS}

H. SAMELSON

Stanford University

Stanford, California

J. P. JaNs

University of Washington

Seattle, Washington 98105

\section{EDITORS}

J. DugundJI

University of Southern California Los Angeles, California 90007

RICHARD ARENS

University of California

Los Angeles, California 90024

\section{ASSOCIATE EDITORS}

E. F. BECKENBACH

B. H. NEUMANN

F. WOLF

K. YosidA

\section{SUPPORTING INSTITUTIONS}

UNIVERSITY OF BRITISH COLUMBIA

CALIFORNIA INSTITUTE OF TECHNOLOGY

UNIVERSITY OF CALIFORNIA

MONTANA STATE UNIVERSITY

UNIVERSITY OF NEVADA

NEW MEXICO STATE UNIVERSITY

OREGON STATE UNIVERSITY

UNIVERSITY OF OREGON

OSAKA UNIVERSITY

UNIVERSITY OF SOUTHERN CALIFORNIA
STANFORD UNIVERSITY

UNIVERSITY OF TOKYO

UNIVERSITY OF UTAH

WASHINGTON STATE UNIVERSITY

UNIVERSITY OF WASHINGTON

AMERICAN MATHEMATICAL SOCIETY CHEVRON RESEARCH CORPORATION TRW SYSTEMS

NAVAL ORDNANCE TEST STATION 


\section{Pacific Journal of Mathematics}

\section{Vol. 20, No. $1 \quad$ September, 1967}

Leonard Daniel Baumert, Extreme copositive quadratic forms. II ........ 1

Edward Lee Bethel, A note on continuous collections of disjoint

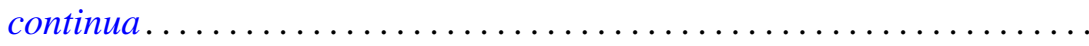

Delmar L. Boyer and Adolf G. Mader, A representation theorem for abelian groups with no elements of infinite p-height ...................

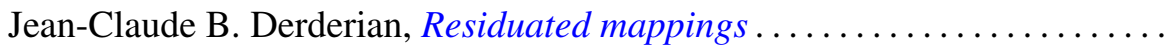

Burton I. Fein, Representations of direct products of finite groups ......... 45

John Brady Garnett, A topological characterization of Gleason parts.......

Herbert Meyer Kamowitz, On operators whose spectrum lies on a circle or

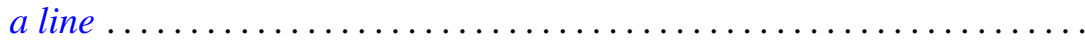

Ignacy I. Kotlarski, On characterizing the gamma and the normal distribution ........................................

Yu-Lee Lee, Topologies with the same class of homeomorphisms ..........

Moshe Mangad, Asymptotic expansions of Fourier transforms and discrete polyharmonic Green's functions ...........................

Jürg Thomas Marti, On integro-differential equations in Banach spaces ....

Walter Philipp, Some metrical theorems in number theory.............. 109

Maxwell Alexander Rosenlicht, Another proof of a theorem on rational

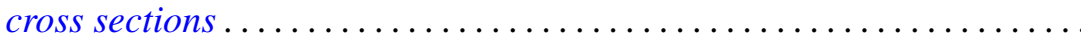

Kenneth Allen Ross and Karl Robert Stromberg, Jessen's theorem on Riemann sums for locally compact groups

Stephen Simons, A theorem on lattice ordered groups, results of Ptak, Namioka and Banach, and a front-ended proof of Lebesgue's theorem...

Morton Lincoln Slater, On the equation $\varphi(x)=\int_{x} x+1 K(\xi) f[\varphi(\xi)] d \xi \ldots$ 155 Arthur William John Stoddart, Existence of optimal controls .. 167 Burnett Roland Toskey, A system of canonical forms for rings on a direct sum of two infinite cyclic groups ....................

Jerry Eugene Vaughan, A modification of Morita's characterization of dimension 